\title{
Influence of aerobic training on neurohormonal and hemodynamic responses to head-up tilt test and on autonomic nervous activity at rest and after exercise in patients after bypass surgery
}

Maria Bilińska ${ }^{1}$, Magdalena Kosydar-Piechna ${ }^{2}$, Tomasz Mikulski ${ }^{4}$, Ewa Piotrowicz ${ }^{2}$, Anna Gąsiorowska ${ }^{4}$, Walerian Piotrowski ${ }^{3}$, Krystyna Nazar ${ }^{4}$, Ryszard Piotrowicz ${ }^{2}$

${ }^{1}$ Department of Cardiac Arrhythmia, Institute of Cardiology, Warsaw, Poland

${ }^{2}$ Department of Cardiac Rehabilitation and Noninvasive Electrocardiology, Institute of Cardiology, Warsaw, Poland

${ }^{3}$ Department of Epidemiology, Cardiovascular Disease Prevention and Health Promotion, Institute of Cardiology, Warsaw, Poland

${ }^{4}$ Department of Applied Physiology, Mossakowski Medical Research Centre,

Polish Academy of Sciences, Warsaw, Poland

\begin{abstract}
Background: Little is known about the influence of aerobic training on the neurohormonal and hemodynamic responses to head-up tilt (HUT) and on autonomic balance at rest and after exercise in optimally treated, low risk post-coronary artery bypass grafting (CABG) patients.
\end{abstract}

Methods: One hundred male patients, mean age $56 \pm 6$ years, 3 months after $C A B G$, were randomized to either 6-week training on cycloergometer, 3 times a week, at 70-80\% of max tolerated heart rate (HR) (training group, $n=50)$ or to a control group $(n=50)$. At baseline and at the end of the study, all patients underwent: (1) cardiopulmonary exercise test with HR recovery (HRR) assessment; (2) 60\% HUT during which HR, blood pressure (BP), stroke volume (SV by impedance cardiography) were monitored and blood samples were taken for determination of plasma catecholamines and ANP levels, and plasma renin activity; (3) assessment of HR variability (HRV) in the time and frequency domains at rest.

Results: During the final tests, HUT-induced changes in HR, BP, SV, cardiac output, total peripheral resistance, and noradrenaline were significantly lower in training group than in controls. In addition, after training faster post-exercise HRR, increased SDNN and a tendency towards an increase in the high frequency HRV power spectrum were found.

Conclusions: Aerobic training improved neurohormonal and hemodynamic responses to head-up tilt test and favorably modified sympatho-vagal balance in low risk post-CABG patients. (Cardiol J 2013; 20, 1: 17-24)

Key words: exercise training, head-up tilt test, autonomic balance

Address for correspondence: Maria Bilińska, MD, PhD, Department of Cardiac Arrhythmia, Institute of Cardiology, ul. Alpejska 42, 04-628 Warszawa, Poland, tel: +48 22 3434417, fax: +48 22 3434520, e-mail: mbilinska@ikard.pl

Received: 20.08.2012 Accepted: 08.10.2012 


\section{Introduction}

Among mechanisms responsible for the favourable impact of exercise training on the outcome in patients with coronary artery disease (CAD) could be an improvement in the autonomic nervous system function.

To date, only very few prospective studies have assessed the influence of exercise training on sympatho-vagal balance in patients after coronary artery bypass grafting (CABG), but not in low risk population, using either heart rate variability (HRV) analysis during Holter monitoring or heart rate recovery (HRR) measured immediately after exercise [1-6]. Moreover, there are no data on the effect of short-term aerobic training in patients after CABG on neurohormonal and hemodynamic responses to an orthostatic stress, such as head-up tilt table test (HUT), which is thought to increase the sympathetic nervous system activity [7].

Therefore, the aim of our study was to evaluate the influence of aerobic training on the neurohormonal and hemodynamic responses to HUT and on sympato-vagal balance at rest and after exercise in low risk, optimally treated post-CABG patients.

\section{Methods}

One hundred twenty male patients with stable CAD who during 2005-2008 underwent off-pump CABG were considered eligible for the study (Table 1).

The inclusion criteria were: $\mathrm{CABG}>3$ months before recruitment, clinical stability for at least 2 months before the entry to the study, preserved left ventricular (LV) function (ejection fraction $\mathrm{EF}>50 \%)$. The exclusion criteria were: unstable angina ( $<3$ months), congestive heart failure, uncontrolled hypertension, cardiac rhythm disturbances, chronotropic incompetence, history of vasovagal syncope, diabetes mellitus, chronic obstructive pulmonary disease, peripheral vascular disease, impaired renal or hepatic function, chronic inflammatory disease, chronic treatment with nitrate and/or diuretics, lack of patient's consent.

Table 1. Baseline clinical characteristics of the study groups.

\begin{tabular}{|c|c|c|c|}
\hline Characteristics & Control $(n=50)$ & Trained $(n=50)$ & $\mathbf{P}$ \\
\hline Mean age [years] & $56 \pm 6$ & $57 \pm 6$ & NS \\
\hline Smoking history & $28(56 \%)$ & $25(50 \%)$ & NS \\
\hline Current smokers & 0 & 0 & NS \\
\hline Hypertension & $22(44 \%)$ & $24(48 \%)$ & NS \\
\hline Body mass index $\left[\mathrm{kg} / \mathrm{m}^{2}\right]$ & $27.3 \pm 3.1$ & $27.6 \pm 2.3$ & NS \\
\hline History of angina [months] & $43.5 \pm 32$ & $44.7 \pm 39$ & NS \\
\hline Post CABG period [weeks] & $16.4 \pm 0.8$ & $17.1 \pm 1.1$ & NS \\
\hline \multicolumn{4}{|l|}{ Vessel disease: } \\
\hline 1-vessel & $4(8 \%)$ & $5(10 \%)$ & NS \\
\hline 2-vessel & $12(24 \%)$ & $10(20 \%)$ & NS \\
\hline 3-vessel & $34(68 \%)$ & $35(70 \%)$ & NS \\
\hline Glucose $[\mathrm{mmol} / \mathrm{L}]$ & $5.2 \pm 0.7$ & $5.1 \pm 0.7$ & NS \\
\hline Total-C [mmol/L] & $4.1 \pm 0.9$ & $3.8 \pm 0.75$ & NS \\
\hline HDL-C [mmol/L] & $1.23 \pm 0.26$ & $1.22 \pm 0.26$ & NS \\
\hline Triglycerides $[\mathrm{mmol} / \mathrm{L}]$ & $1.44 \pm 0.76$ & $1.35 \pm 0.59$ & NS \\
\hline LVEF [\%] & $56.3 \pm 8.8$ & $57.1 \pm 8.9$ & NS \\
\hline Physical capacity $\left(\right.$ peak $\left.\mathrm{VO}_{2}\right)[\mathrm{mL} / \mathrm{kg} / \mathrm{min}]$ & $24.3 \pm 4.0$ & $25.1 \pm 3.6$ & NS \\
\hline \multicolumn{4}{|l|}{ Medications (\%): } \\
\hline Beta-blocker & 100 & 100 & NS \\
\hline ACE inhibitor & 100 & 100 & NS \\
\hline Calcium channel blocker & 88 & 88 & NS \\
\hline Statins & 100 & 100 & NS \\
\hline Aspirin & 100 & 100 & NS \\
\hline
\end{tabular}

All values are presented as mean \pm SD or percentages of the number of patients in each group; NS - non significant; CABG - coronary artery bypass grafting; $\mathrm{C}$ - cholesterol; LVEF — left ventricular ejection fraction; peak $\mathrm{VO}_{2}$ — peak oxygen uptake; ACE — angiotensin-converting enzyme 
All patients were in sinus rhythm and agreed to maintain a stable level of physical activity during the study. Medications were not altered within 4 weeks prior to the enrollment and throughout the study.

The Institutional Ethics Committee approved the study protocol, and an informed written consent was obtained from each patient.

\section{Study design}

At entry, eligible patients underwent physical examination, ECG, 2-dimensional ECHO, cardiopulmonary exercise test (CPET) with gas exchange measurements, 24-h Holter monitoring (24-Holter), passive HUT and blood sampling for laboratory analyses.

Then, they were randomized either to a 6 -week aerobic training program (training group) or to a control group, and the determinations such as CPET, 24-Holter and HUT were repeated at the conclusion of 6 -week study protocol.

\section{Biochemical measurements}

Blood samples were taken for biochemical tests between 8 and 9 a.m. after an overnight fast and routine measurements (e.g. blood glucose, lipid profile) were performed immediately using standard laboratory techniques. In addition, plasma concentrations of noradrenaline (NA), adrenaline (A), plasma renin activity (PRA) and atrial natriuretic peptide (ANP) were determined immediately after each HUT.

To measure plasma levels of NA, A, PRA and ANP, blood was drawn into vacutainers containing EDTA. Plasma was immediately separated by centrifugation at $2000 \times \mathrm{g}$ for $10 \mathrm{~min}$ at $4^{\circ} \mathrm{C}$ and the samples were frozen immediately and stored at $-70^{\circ} \mathrm{C}$ until analysis.

Plasma concentrations of hormones were determined by radioimmunoassay used as follows: tests produced by BioSource Europe S.A., Belgium (2 CAT RIA) for NA and A, CIS bio international kit (France) for ANP and Immunotech Angiotensin I Kit (Czech Republic) for PRA.

\section{Echocardiography}

At baseline, ECHO-2D was performed to determine LV end-diastolic and end-systolic volumes using a modified Simpson's rule, from which LVEF (\%) was automatically calculated.

\section{Cardiopulmonary exercise stress test}

Details of the CPET protocol have been previously described [8]. Briefly, all patients underwent symptom-limited CPET according to a modified
Bruce protocol, using a Schiller treadmill (Carrollton, USA) with gas exchange analysis (600USB CPX, ZAN Messgeräte GmbH, Germany).

The following variables were assessed: exercise duration [min], heart rate (HR) [bpm] at rest and at peak exercise, double product i.e. product of $\mathrm{HR}$ and systolic $\mathrm{BP}[\mathrm{mm} \mathrm{Hg} / \mathrm{min}]$ at rest and at peak exercise, and peak oxygen uptake (peak $\left.\mathrm{VO}_{2}\right)[\mathrm{mL} /$ $/ \mathrm{kg} / \mathrm{min}]$.

In addition, HRR, i.e. the difference between HR at peak effort and that measured in the first (HRR-1) and second minutes (HRR-2) of the recovery period, was assessed.

\section{4-hour Holter monitoring with HRV analysis}

24-h ECG was recorded with a Spacelabs Lifecard system (Spacelabs, Del Mar Reynolds, Hertford, UK). Each recording began with a 15-min rest in the supine position followed by 15 min of standing. The standard ECG analysis included assessment of basal HR, occurrence of ventricular or supraventricular arrhythmias, ST segment depression and atrioventricular blocks.

HRV indices were calculated after a prospective interactive ECG analysis, using the Impresario Space software (Del Mar Reynolds). The measurements of HRV included time domain and frequency domain indices. Within the time domain, HR and standard deviation (SD) of all normal RR intervals (SDNN) [ms] were assessed. The frequency domain indices were calculated after fast Fourier transformation of five 5-minute ECG segments, recorded between 2 a.m. and 6 a.m. The highest and lowest values were excluded, and the 3 remaining values were averaged. The power spectrum density of low frequency (LF): 0.04-0.15 Hz, high frequency (HF): $0.15-0.4 \mathrm{~Hz}$, and the low frequency to high frequency ratio $(\mathrm{LF} / \mathrm{HF})$ were calculated.

\section{Head-up tilt table test}

After a 30-min supine equilibration period, an upright posture with tilt angle of 60-degrees for 10 min was applied. During the test the following variables were monitored: HR during continuously recorded ECG, BP obtained with mercury sphygmomanometer, and stroke volume (SV), which was determined by impedance cardiography. As described previously, the system allows for the off-line, beat-to-beat evaluation of SV and HR [8-10]. Cardiac output (CO) was calculated as a product of SV and HR. Total peripheral resistance (TPR) was calculated by dividing mean $\mathrm{BP}$ by $\mathrm{CO}$. Before and after the test, blood samples for plasma concentrations of NA, $\mathrm{A}, \mathrm{PRA}$ and $\mathrm{ANP}$ were taken from the antecubital vein. 
Table 2. Effect of exercise training on plasma hormone levels in both study groups.

\begin{tabular}{|c|c|c|c|c|c|c|}
\hline \multirow[t]{2}{*}{ Variables } & \multicolumn{2}{|c|}{ Control $(n=50)$} & \multirow[t]{2}{*}{$\mathbf{P}$} & \multicolumn{2}{|c|}{ Training $(n=50)$} & \multirow[t]{2}{*}{$\mathbf{P}$} \\
\hline & Before & After & & Before & After & \\
\hline Noradrenaline [pmol/mL] & $4.41 \pm 0.34$ & $4.67 \pm 0.26$ & NS & $4.39 \pm 0.25$ & $4.33 \pm 0.26$ & NS \\
\hline Adrenaline $[\mathrm{pmol} / \mathrm{mL}]$ & $0.35 \pm 0.04$ & $0.31 \pm 0.05$ & NS & $0.33 \pm 0.04$ & $0.35 \pm 0.04$ & NS \\
\hline Plasma renin activity [ng/mL/h] & $0.96 \pm 0.11$ & $0.93 \pm 0.12$ & NS & $0.96 \pm 0.13$ & $0.98 \pm 0.14$ & NS \\
\hline Atrial natriuretic peptide $[\mathrm{pg} / \mathrm{mL}]$ & $42.1 \pm 7.5$ & $40.8 \pm 6.2$ & NS & $38.9 \pm 4.9$ & $41.0 \pm 5.0$ & NS \\
\hline
\end{tabular}

All values are presented as mean $\pm \mathrm{SD}$; NS - non significant

Table 3. Results of the $24 \mathrm{~h}$ Holter monitoring with selected HRV measures in both study groups.

\begin{tabular}{|c|c|c|c|c|c|c|}
\hline & \multicolumn{2}{|c|}{ Control $(n=50)$} & \multirow[t]{2}{*}{$\mathbf{P}$} & \multicolumn{2}{|c|}{ Training $(n=50)$} & \multirow[t]{2}{*}{$\mathbf{P}$} \\
\hline & Before & After & & Before & After & \\
\hline Average HR [/min] & $67.8 \pm 7.0$ & $61.9 \pm 7.2$ & NS & $69.1 \pm 7.7$ & $66.5 \pm 7.8$ & $<0.05$ \\
\hline Min. HR [/min] & $53.0 \pm 13.2$ & $51.1 \pm 6.8$ & NS & $53.5 \pm 10.6$ & $50.1 \pm 6.9$ & $<0.05$ \\
\hline Max. HR [/min] & $104.3 \pm 14.9$ & $101.2 \pm 12.2$ & NS & $105.9 \pm 15.1$ & $102.8 \pm 16.9$ & 0.07 \\
\hline SDNN total [ms] & $119.7 \pm 42.6$ & $122.3 \pm 46.3$ & NS & $122.8 \pm 42.2$ & $136.3 \pm 49.2^{*}$ & $<0.05$ \\
\hline $\mathrm{LF}\left[\mathrm{ms}^{2} / \mathrm{Hz}\right]$ & $284 \pm 257$ & $306 \pm 365$ & NS & $288 \pm 268$ & $274 \pm 263$ & NS \\
\hline $\mathrm{HF}\left[\mathrm{ms}^{2} / \mathrm{Hz}\right]$ & $174 \pm 144$ & $186 \pm 174$ & NS & $181 \pm 157$ & $227 \pm 173$ & 0.07 \\
\hline LF/HF & $1.9 \pm 1.4$ & $2.1 \pm 1.5$ & NS & $2.0 \pm 1.5$ & $1.7 \pm 1.3$ & 0.09 \\
\hline
\end{tabular}

Data for SDNN, LF, HF and LF/HF were logarithmically transformed before analysis, however, only the original untransformed values are presented in the Table. All values are presented as mean \pm SD; NS - non significant; HR - heart rate; SDNN - standard deviation of all normal R wave to R wave (N-N) intervals; LF, HF - low and high frequency spectral components of heart rate variability (HRV); ${ }^{*} p<0.05$ changes of variables between groups

\section{Training program}

The program consisted of 60 -min bicycle ergometer exercise sessions 3 times per week for 6 weeks. The exercise loads were applied in an interval manner, i.e. 4-min exercise bouts separated by 2 -min rests in between with gradually increased workload until $70-80 \%$ of maximal HR achieved during CPET was reached.

Control patients were instructed to maintain their habitual activities.

\section{Statistical analysis}

Continuous variables were tested for normal distribution with the Shapiro-Wilk test and were expressed as mean \pm SD if normally distributed or as percentages. Variables were compared using Student's t-test or analysis of variance for normally distributed variables, and for other continuous variables the Kruskal-Wallis test was used, followed either by the Mann-Whitney or the Wilcoxon rank test, as appropriate. Comparisons for the discrete variables between groups were performed using $\chi^{2}$ test. Correlations were determined with Pearson's or Spearman's correlation test as appropriate. $P$ value $<0.05$ was considered significant.
The analysis was performed using SAS statistical software (version 8.02; Cary, North Carolina, USA).

\section{Results}

The study comprised male subjects with a similar history of angina and a similar clinical status.

Of 120 clinically eligible patients, 20 patients were excluded from the study (10 in each group) because of administrative reasons. There were no differences between the two study groups in regards to baseline clinical characteristics (Table 1).

As evidenced in Table 2, no significant differences existed between the two groups in the baseline values of A, NA, PRA and ANP measured at the beginning and at the end of the study.

The findings of 24-Holter are shown in Table 3. After a 6 -week period, only in the training group did minimum and average HR decrease significantly. Moreover, SDNN as another index of the time domain increased significantly in trained patients comparing with controls. For the frequency domain measures, although in the control group no significant changes were found between baseline variables and those assessed after 6 weeks, a tendency to- 
Table 4. Results of the cardiopulmonary exercise tests in the study groups.

\begin{tabular}{|c|c|c|c|c|c|c|}
\hline & \multicolumn{2}{|c|}{ Control $(n=50)$} & \multirow[t]{2}{*}{$\mathbf{P}$} & \multicolumn{2}{|c|}{ Training $(n=50)$} & \multirow[t]{2}{*}{$\mathbf{P}$} \\
\hline & Before & After & & Before & After & \\
\hline Duration [min] & $12.9 \pm 1.6$ & $13.3 \pm 1.9$ & NS & $12.5 \pm 1.8$ & $14.1 \pm 1.6^{*}$ & $<0.05$ \\
\hline Peak $\mathrm{VO}_{2}[\mathrm{~mL} / \mathrm{kg} / \mathrm{min}]$ & $24.3 \pm 4.0$ & $25.7 \pm 4.9$ & NS & $24.9 \pm 3.6$ & $27.3 \pm 4.5^{*}$ & $<0.05$ \\
\hline $\mathrm{HR}$ at rest [/min] & $65.6 \pm 9.7$ & $65.2 \pm 8.2$ & NS & $67.4 \pm 10.9$ & $63.8 \pm 10.2^{*}$ & $<0.05$ \\
\hline Peak HR [/min] & $122.8 \pm 15.9$ & $126.3 \pm 15.2$ & NS & $123.0 \pm 14.8$ & $128.3 \pm 15.7$ & NS \\
\hline $\begin{array}{l}\mathrm{DP} \text { at rest }[\mathrm{mm} \mathrm{Hg} / \\
/ \mathrm{min} \times 100]\end{array}$ & $78.2 \pm 15.1$ & $77.9 \pm 13.3$ & NS & $79.1 \pm 14.5$ & $76.9 \pm 13.9$ & NS \\
\hline $\begin{array}{l}\text { peak DP }[\mathrm{mm} \mathrm{Hg} / \\
/ \mathrm{min} \times 100]\end{array}$ & $207.4 \pm 47.3$ & $214.3 \pm 44.2$ & NS & $209.5 \pm 44.8$ & $216.9 \pm 45.7$ & NS \\
\hline HRR-1 [/min] & $19.9 \pm 8.2$ & $18.9 \pm 9.5$ & NS & $19.5 \pm 7.8$ & $24.6 \pm 9.1 \#$ & $<0.05$ \\
\hline HRR-2 [/min] & $39.8 \pm 8.9$ & $39.7 \pm 8.4$ & NS & $38.8 \pm 9.6$ & $43.5 \pm 10.1^{*}$ & $<0,05$ \\
\hline
\end{tabular}

All values are presented as mean $\pm \mathrm{SD} ; \mathrm{NS}$, non significant; peak $\mathrm{VO}_{2}$ - peak oxygen uptake; $\mathrm{HR}$ - heart rate; peak $\mathrm{HR}$ - $\mathrm{HR}$ at peak exercise; $\mathrm{DP}$ - double product, that is, product of HR and systolic blood pressure; peak DP — DP at peak exercise; HRR-1, HRR-2 - heart rate recovery in the first and second minute after ending exercise test; ${ }^{*} p<0.05, \# p<0.02$, changes of variables between groups

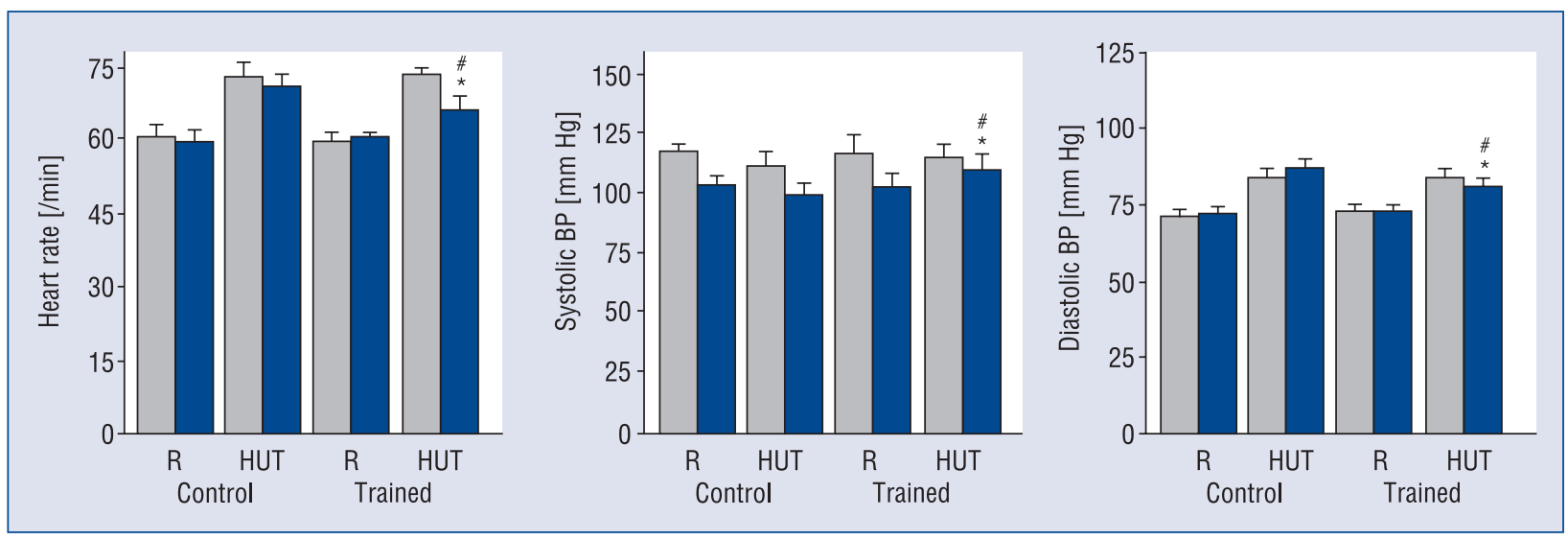

Figure 1. Heart rate and blood pressure (BP) at rest (R) and at the end of head-up tilt test (HUT) in the control and training groups. Values are means $\pm \mathrm{SD}$; grey bars — initial tests; blue bars — final tests (after 6 weeks); ${ }^{*} p<0.05$ — significant differences between values obtained before and at the end of HUT during the final test within each group; ${ }^{*} \mathrm{p}<0.05$ - significant differences between groups during the final test.

wards an increase in $\mathrm{HF}$ and decrease in $\mathrm{LF} / \mathrm{HF}$ was observed in the training group.

Moreover, even though no significant differences were found between trained patients and controls in mean delta values of $\mathrm{LF}(\Delta=-13.7 \pm 186$ vs. $21.9 \pm 206.3$, respectively $), \operatorname{HF}(\Delta=-33.8 \pm 157.6$ vs. $14.6 \pm 127.4$, respectively) and $\mathrm{LF} / \mathrm{HF}(\Delta=$ $=-0.41 \pm 1.6$ vs. $0.34 \pm 1.9$, respectively), they shifted in the opposite direction.

Table 4 demonstrates CPET data in patients studied. Exercise duration, resting $\mathrm{HR}$ and the objective index of physical capacity, e.g., peak $\mathrm{VO}_{2}$ improved significantly only in the training group. Moreover, a 6-week training program resulted in a significant improvement in such variables of parasympathetic activity as HRR- 1 and HRR-2 (21\% vs. $12 \%$, respectively, $\mathrm{p}<0.05)$ in trained patients comparing with controls.

Hemodynamic data during HUT are shown in Figure 1 . There were no significant differences in any analyzed cardiovascular indices before initial tests between the two groups. The HUT caused increases in HR and diastolic BP and decreases in systolic BP in both groups, however, these changes were significantly smaller at the end of final tests only in the training group. Importantly, in the training group the decreases in SV and CO during the final tests were smaller than in the control group (by 


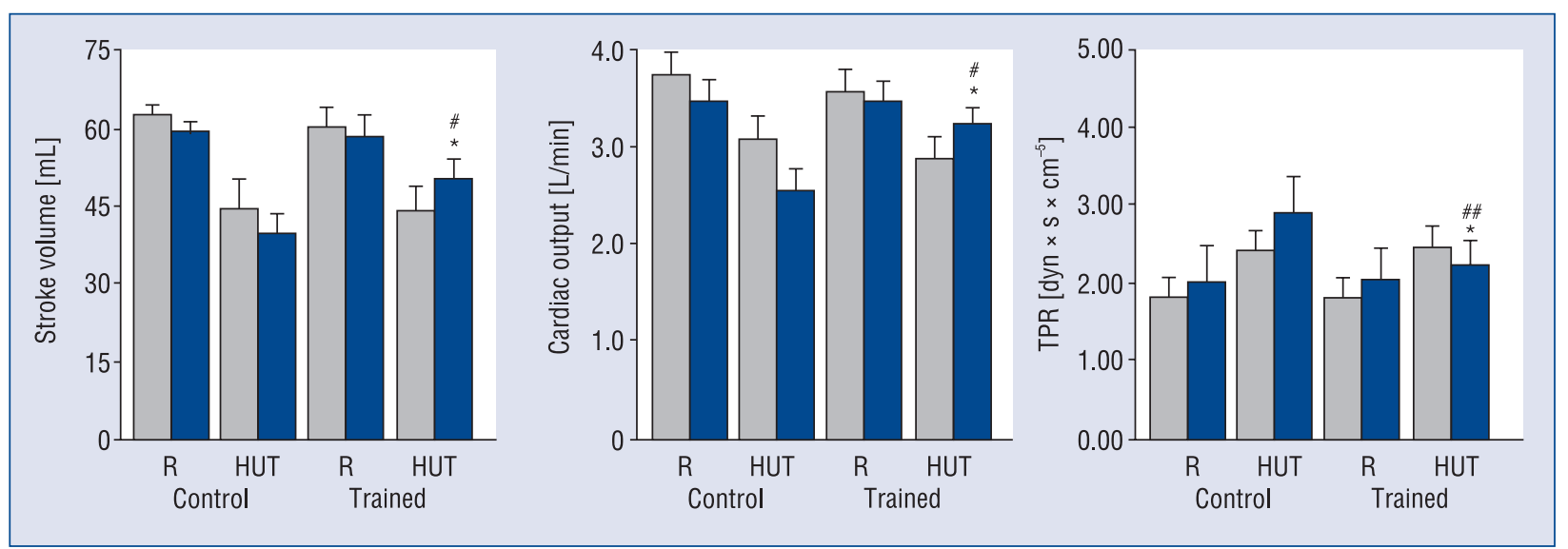

Figure 2. Stroke volume, cardiac output and total peripheral resistance (TPR) at rest (R) and at the end of head-up tilt test (HUT) in the control and training group. Values are means \pm SD; grey bars — initial tests; blue bars - the final tests (after 6 weeks); TPR is calculated as mean blood pressure [mm Hg] $\times 79.9 /$ cadiac output [L/min], where 79.9 is the conversion factor to dyn $\times \mathrm{s} \times \mathrm{cm}^{-5} ;{ }^{*} \mathrm{p}<0.05$, significant differences between values obtained before and at the end of HUT during the final test within each group; ${ }^{\#} p<0.05,{ }^{\# \#} p<0.01$, significant differences between groups during the final test.

$15 \%$ and $14 \%$, respectively, $\mathrm{p}<0.05$ ) (Fig. 2). Moreover, the HUT-induced increases in TPR during the final tests were significantly smaller in trained patients than in controls ( $12 \%$ vs. $24 \%, \mathrm{p}<0.01)$.

Figure 3 demonstrates plasma hormone concentrations at rest and at the end of HUT in both study groups. There were no significant differences between the two groups in the resting plasma levels of A, NA, PRA and ANP.

Although the orthostatic stress caused significant increases in concentrations of NA and PRA at the end of the first HUT in both study groups, at the end of the study, the plasma NA level changed in the opposite direction, e.g., decreased in trained patients and increased in controls $(-7 \%$ vs. $23 \%, \mathrm{p}<0.01)$. The changes in the remaining hormones did not differ significantly between the groups. There were no correlations between the HUT-induced changes in the plasma neurohormones (NA, A, PRA, ANP) and delta hemodynamic parameters (HR, systolic and diastolic $\mathrm{BP}, \mathrm{SV}, \mathrm{CO}, \mathrm{TPR})$ between trained and untrained post-CABG patients, and in the whole group.

The health status was stable in all patients during the study, and in none patient were there any adverse effects of exercise training observed. Moreover, none of the patients experienced presyncope during HUT.

\section{Discussion}

This study provides a comprehensive insight into autonomic control of cardiovascular (CV) sys- tem function in low risk post-CABG patients who underwent a short-term exercise training.

Our results strongly suggest that a 6 -week aerobic training on cycloergometer in patients with stable angina and preserved LV function improved not only their functional capacity as evidenced by a significant increase of the peak $\mathrm{VO}_{2}$, but also decreased sympathetic nervous activity measured during daily life and after provocation.

We used noninvasive techniques based on ECG as indicators of such autonomic nervous system activities as HR and HRV, which are thought to reflect tonic baseline autonomic function, and HRR, which primarily represents reflex vagal reactivity after exercise stress test. Moreover, for the first time the capacity of the CV to respond to sympathetic stimulation during orthostatic stress such as passive HUT was assessed. The assumption of upright posture leads to displacement of blood from the upper to the lower body parts mainly to legs. As a consequence, central venous pressure and SV decrease, and HR increases secondary to vagal withdrawal and sympathetic stimulation (via baroreceptors) [7].

In our study, the magnitude of sympathetic nervous activity during HUT performed at the end of the study was significantly lower in the training group compared with controls.

We found that HUT-induced changes in HR, systolic and diastolic BP were significantly smaller at the end of final tests in trained patients than in controls. Moreover, only in the training group were 


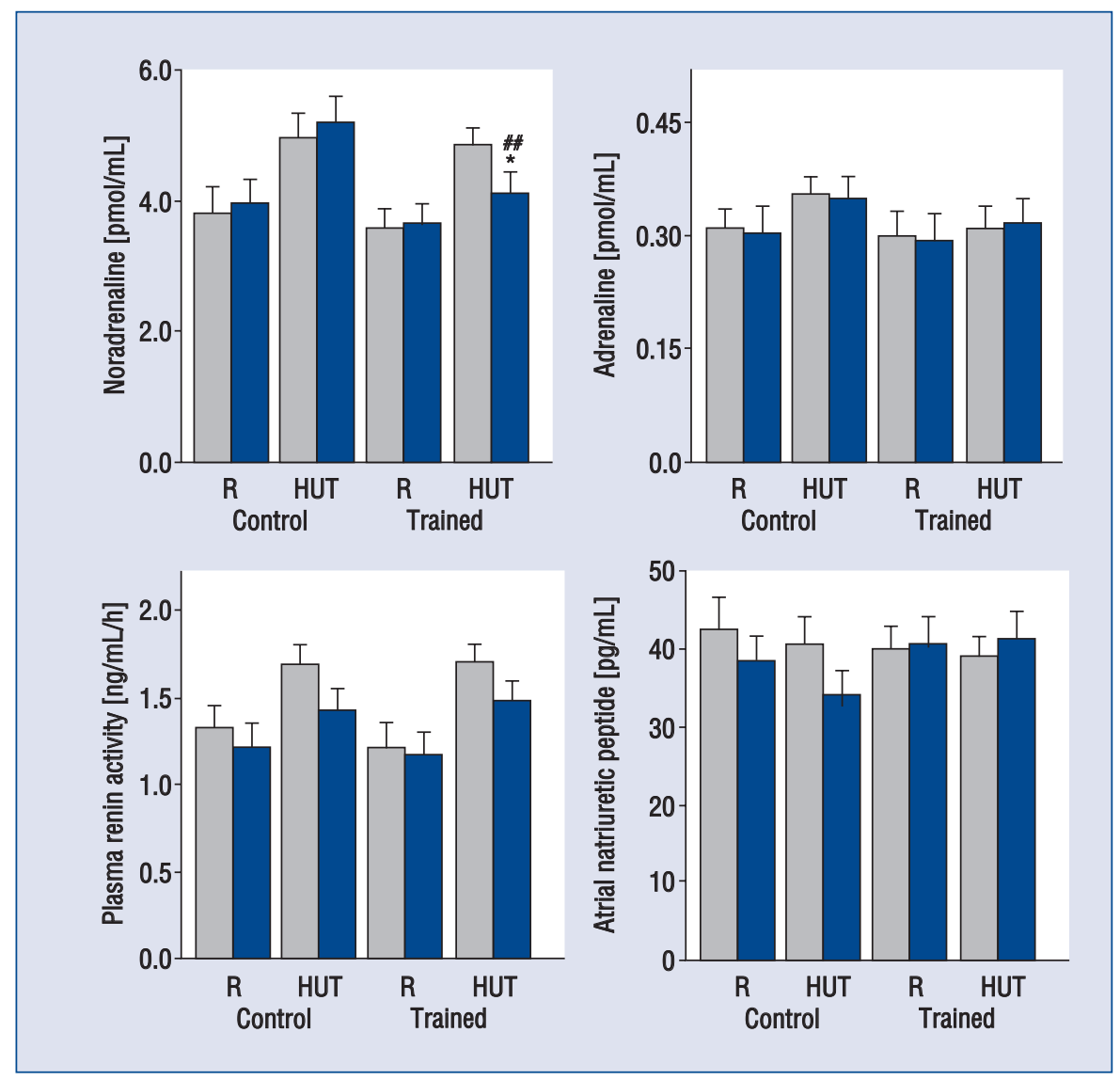

Figure 3. Plasma catecholamine and atrial natriuretic peptide concentrations, and plasma renin activity at rest (R) and at the end of head-up tilt test (HUT) in the control and training group. Values are means \pm SD; grey bars - initial tests; blue bars - the final tests (after 6 weeks); ${ }^{*} p<0.05$ - significant differences between values obtained before and at the end of HUT during the final test within each group; ${ }^{\# \#} p<0.01-$ significant differences between groups during the final test.

the decreases in SV and CO smaller during the final tests.

Furthermore, the HUT-induced increases in TPR and NA during the final tests were significantly smaller in trained patients than in controls. Thus, even a short-term training was able to decrease sympathetic activity and improve $\mathrm{CV}$ responses after changing from supine to upright posture.

The mechanism of this favorable neurohormonal response to HUT in trained patients is unclear. We did not find any significant correlations between delta neurohormones and delta hemodynamic indices in trained versus untrained post-CABG patients. We can only hypothesize that exercise therapy contributed to better tolerance of orthostatic stress not only by peripheral adaptation but also by improving cardiac functions.

The heart-rate profile during the standard exercise test has been shown to predict all-cause mortality and cardiac events in patients with known or suspected CV diseases [11-13]. Recently, it has been reported that exercise training may improve HRR in CAD patients [1, 2]. However, only very few small studies have been conducted in post-CABG patients and shown that HRR improved after exercise training $[14,15]$. Our results confirmed that HRR measured not only in the first but also in the second minute immediately after CPET improved significantly only in trained post-CABG patients. This suggest that HRR as a marker of reflex autonomic activity was modifiable even after a 6 -week supervised exercise training. Moreover, since HRR is also related to peak HR, in patients without chronotropic incompetence, it could be an expression of physical capacity, as was the case in our trained patients.

Since impaired autonomic function, mainly decreased parasympathetic activity, has been reported to occur after CABG, we performed the first measurement of HRV after 3 months and did not find any differences between the training group and 
controls. It is noteworthy that after a 6 -week exercise training such time domain indices as minimum and average HR, and SDNN improved only in trained patients. For the frequency domain measures, a tendency towards an increase in $\mathrm{HF}$ and decrease in LF/ /HF were noted only in the training group.

Moreover, mean delta values of LF, $\mathrm{HF}$ and LF/ /HF shifted in the opposite direction despite the lack of significant differences between the study groups, suggesting a greater than in controls modulatory effect of exercise training on the sinus node.

To date, only very few studies have been conducted in post-CABG patients, but not in low risk population $[1,3,4]$. Despite the fact that researchers included only a small number of patients who participated in different training programs starting in the early postoperative period, they generally reported that exercise therapy positively modified HRV in post-CABG patients. Although the mechanism of the beneficial effect of exercise training on $\mathrm{HRV}$ is not clear, the findings to date suggest a shift of the sympatho-vagal balance toward parasympathetic dominance.

It is noteworthy that despite the fact that resting values of catecholamines did not differ between trained patients and controls over the study period, an improvement in parameters of autonomic function was observed only in the training group.

To the best of our knowledge, this is the first study to show that a 6 -week aerobic training on cycloergometer in post-CABG patients with preserved LV function, who are already under optimal treatment, had the potential to improve not only their physical capacity but neurohormonal and hemodynamic responses to orthostatic stress. Moreover, a short-term training appeared to be an effective intervention able to improve cardiac autonomic regulation during daily life and after exercise.

Given that both autonomic balance and physical capacity are known to have an impact on prognosis, individually programmed training sessions should be advised to all CAD patients, especially those after CABG.

\section{Limitations of the study}

Firstly, the results of this study can be applied to a specific group of male patients after $\mathrm{CABG}$ and at low prognostic risk.

Secondly, the medical therapy included betablockers and angiotensin-converting enzyme inhibitors, which could affect markers of autonomic function. Moreover, a potentially beneficial interaction between exercise training and antiadrenergic therapy might occur.
Thirdly, cardiac function during HUT was determined only non-invasively by an automatized impedance cardiography method, which allows for automatic evaluation of SV and HR.

Aside from the study limitations listed above, the lack of determination of blood and plasma density during orthostasis is one that has to be considered.

\section{Conclusions}

The study demonstrated that a 6 -week aerobic training in low risk post-CABG patients with preserved LV function, improved neurohormonal and hemodynamic responses to orthostatic stress and favorably modified sympatho-vagal balance during daily life and after exercise.

\section{Acknowledgements}

This work was supported by the grant $\mathrm{N}^{\circ} 2$ P05D 07428 from the Ministry of Science and Higher Education, Poland.

Conflict of interest: none declared

\section{References}

1. Iellamo F, Legramante JM, Massaro M, Raimondi G, Galante A. Effects of residential exercise training on baroreflex sensitivity and heart rate variability in patients with coronary artery disease. Circulation, 2000; 102 2588-2592.

2. La Rovere MT, Bersano C, Gnemmi M, Specchia G, Schwart PJ. Exerciseinduced increase in baroreflex sensitivity predicts improved prognosis after myocardial infarction. Circulation, 2002; 106: 945-949.

3. Lucini D, Milani RV, Constantino G, Lavie CJ, Porta A, Pagani M. Effects of cardiac rehabilitation and exercise training on autonomic regulation in patients with coronary artery disease. Am Heart J, 2002; 143: 977-983.

4. Takeyama J, Itoh $\mathrm{H}$, Kato $\mathrm{M}$ et al. Effects of physical training on the recovery of the autonomic nervous activity during exercise after coronary artery bypass grafting: Effects of training after CABG. Jpn Circ J, 2000; 64: 809-813.

5. Tiukinhoy S, Beohar N, Hsie M. Improvement in heart rate recovery after cardiac rehabilitation. J Cardiopulm Rehabil, 2003; 23: 84-87.

6. Adams BJ, Carr JG, Ozonoff Al, Lauer MS, Balady GJ. Effect of exercise training in supervised cardiac rehabilitation programs on prognostic variables from the exercise test tolerance. Am J Cardiol, 2008; 101: 1403-1407.

7. Convertino VA. Lower body negative pressure as a tool for research in aerospace physiology and military medicine. J Grav Physiol, 2001; 8: 1-14.

8. Bilińska M, Kosydar-Piechna M, Gąsiorowska A et al. Influence of dynamic training on hemodynamic, neurohormonal responses to static exercise and inflammatory markers in patients after coronary artery bypass grafting. Circ J, 2010; 74: 2598-2604.

9. Cybulski G. Ambulatory impedance cardiography: new possibilities, J Appl Physiol, 2000; 88: 1509-1510.

10. Cybulski G, Michalak E, Kozluk E, Piatkowska A, Niewiadomski W. Stroke volume and systolic time intervals: beat-to-beat comparison between echocardiography and ambulatory impedance cardiography in supine and tilted positions. Med Biol Eng Comput, 2004; 42: 707-711.

11. Jouven X, Empana JP, Schwartz PJ, Desnos M, Courbon D, Ducimetiere P. Heart-rate profile during exercise as a predictor of sudden death. NEJM 2005; 352: 1951-1958.

12. Myers J, Tan SY, Abella J, Aleti V, Froelicher VF. Comparison of the chronotropic response to exercise and heart rate recovery in predicting cardiovascular mortality. Eur J Cardiovasc Prev Rehabil, 2007; 14: 215-221.

13. Cole CR, Blackstone EH, Pashkow EJ. Heart-rate recovery immediately after exercise as a predictor of mortality. N Engl J Med, 1999; 341: 1351-1357.

14. Kligfield P, McCormick A, Chai A, Jacobson A, Feuerstadt P, Hao SC. Effect of age and gender on heart rate recovery after submaximal exercise during cardiac rehabilitation in patients with angina pectoris, recent acute myocardial infarction, or coronary bypass surgery. Am J Cardiol, 2003; 92: $600-603$.

15. Sato S, Makita S, Majima M. Additional physical activity during cardiac rehabilitation leads to an improved heart rate recovery in male patients after coronary artery bypass grafting. Circ J, 2005; 69: 69-71. 